\title{
Permeabilidade de Solo Laterítico por Diferentes Métodos
}

\section{Permeability of lateritic soil by various methods}

\author{
Tatiana Tavares Rodriguez ${ }^{1}$; Luis Alexandre Weiss², Raquel Souza Teixeira ${ }^{3}$; Carlos José \\ Marques da Costa Branco ${ }^{4}$
}

\begin{abstract}
Resumo
Permeabilidade é uma propriedade de extrema importância para dimensionamento de diferentes tipos de obras de engenharia e é quantificada através do coeficiente de permeabilidade. Apesar do grande uso, ainda existem questionamentos sobre a melhor forma de determinação do coeficiente de permeabilidade. As principais questões são: (1) o tipo de método a ser utilizado e (2) a representatividade de amostras em solos tropicais. Neste contexto, objetivou-se neste trabalho a avaliação da permeabilidade de um solo laterítico, através da comparação de valores de coeficiente de permeabilidade determinados por ensaios in situ e em laboratório. Para tanto, escolheu-se o solo laterítico do Campo Experimental de Engenharia Geotécnica (CEEG) da Universidade Estadual de Londrina (UEL) e quatro equipamentos: permeâmetro de Carga Constante, permeâmetro de Carga Variável, permeâmetro de Guelph e Infiltrômetro. Os resultados mostram que, com exceção do permeâmetro de Carga Constante, todos os métodos apresentaram valor médio de coeficiente de permeabilidade da ordem de $10^{-3} \mathrm{~cm} / \mathrm{s}$ para coeficientes de variação de $37 \%$ a $92 \%$. A heterogeneidade da estrutura do solo laterítico (em macro e microporos) é o provável condicionante da variabilidade encontrada.
\end{abstract}

Palavras-chave: Permeabilidade. Solo Laterítico. Estrutura. Permeâmetros de Laboratório. Guelph e Infiltrômetro.

\begin{abstract}
Soil Permeability is an important property of soil used to dimension several types of engineering works, and it can be quantified by the permeability rates. Despite of the great use, the type of the re doubts about the best way to determine de permeability rates. The main questions are: (1) the type of the method and (2) the reproducibility of samples in tropical soils. So, the objective of this work is to evaluate the permeability of a lateritic soil by comparing values of permeability coefficient determined for laboratory and in situ testing. For this, it was chosen the lateritic soil of Campo Experimental de Engenharia Geotécncia (CEEG) of the Universidade Estadual de Londrina (UEL) and four equipments: constant and variable head permeameters, Guelph permeameter and infiltrometer. The results show that all the methods present mean value of permeability coefficient on the order of $10^{-3} \mathrm{~cm} / \mathrm{s}$ whit variation coefficient in range of $37 \%$ to $92 \%$, except the constant permeameter. The heterogeneous structure of lateritic soil (in macro and micro pores) is the probably determinant of the variability observed.
\end{abstract}

Keywords: Permeability. Lateritic Soil. Structure. Laboratory Permeameters. Guelph Permeameter and Infiltration test.

\footnotetext{
${ }^{1}$ Docente do Dpto. de Transportes e Geotecnia, Universidade Federal de Juiz de Fora; tatiana.rodriguez@ufjf.edu.br

${ }^{2}$ Engenheiro Civil, Londrina, weiss.lu@hotmail.com

${ }^{3}$ Docente do Departamento de Engenharia Civil, Universidade Estadual de Londrina; raquel@uel.br

${ }^{4}$ Docente do Departamento de Engenharia Civil, Universidade Estadual de Londrina; costabranco@uel.br
} 


\section{Solos Lateriticos}

Laterização é um processo acentuado de intemperismo que ocorre frequentemente em regiões tropicais, de alta temperatura e chuvas abundantes, e é caracterizado pela rápida quebra de feldspatos e minerais ferromagnesianos, remoção de sílica e bases e concentração de óxidos de ferro e alumínio (BARATA, 1981; MITCHELL; SITAR, 1982; BIGARELLA; BECKER; PASSOS, 1996).

Solos lateríticos são solos sujeitos à laterização e têm características comuns, como alta porosidade, baixo percentual de silte e agregação de particulas finas, que conduzem esses solos a comportamentos específicos, que a partir da Primeira Conferência Internacional de Solos Tropicais Lateríticos e Saprolíticos (1985), vêm sendo estudados por diversos autores (SANDRONI, 1985; MACCARINI; TEIXEIRA; TRICHÊS, 1989; MASSEY; IRFAN; CIPULLO, 1989; LEROUEIL; VAUGHAN, 1990; BRESSANI; MARTINS; BICA, 1994; MORAES SILVA, 2000; FUTAI, 2002; RODRIGUEZ, 2005; LACERDA, 2010).

Os estudos revelam que o comportamento diferenciado dos solos lateríticos se deve à estrutura (porosidade, agregação e cimentação) e à composição (mineralogia, micro-fissuras, tamanho e forma dos grãos), que afetam a classificação dos solos e as propriedades mecânicas como resistência, deformação e permeabilidade (NOGAMI; VILLIBOR, 1981; VARGAS, 1992; CRUZ, 2004).

Apermeabilidade é a propriedade do solo (meio poroso) que avalia a facilidade de percolação de água através dos seus poros ou vazios. Essa propriedade é quantificada pelo parâmetro conhecido por coeficiente de permeabilidade expresso em unidade de distância por unidade de tempo, e depende de fatores como viscosidade do fluido, granulometria do solo, grau de saturação e estrutura. Cruz (2004) ressalta que o termo condutividade hidráulica tem sido utilizado como sinônimo de permeabilidade apesar do primeiro se aplicar a meio confinado, como no caso de fluxo através de fraturas em rochas.

A estrutura, condicionada pelo arranjo das partículas, define os vazios existentes que são os caminhos possíveis de percolação do fluxo. Em areias puras, utilizadas para filtros e drenos, os vazios são dependentes apenas da compacidade do solo ou índice de vazios (PINTO, 2002). Para outras classes de solos, como solos argilosos lateríticos, a agregação das partículas pode formar, em solos com mesmo índice de vazios, classes diferentes de vazios, denominadas microporos e macroporos, que apresentam comportamento distinto com respeito à permeabilidade, sendo os macroporos os principais responsáveis pela permeabilidade do solo (CRUZ, 2004; MENEZES; RIBEIRO; SAMPAIO, 2006).

A determinação do coeficiente de permeabilidade é o ponto principal na avaliação de fluxo aplicada a obras de estabilidade de encosta; rebaixamento do nível d’água subterrâneo e barragens, além de avaliação de balanço hídrico e escoamento subsuperficial. $\mathrm{O}$ valor do coeficiente de permeabilidade pode ser obtido em campo ou em laboratório por métodos diversos. Em campo, é comum o uso de Matsuo, Permeâmetro de Guelph, Infiltrômetro, Permeâmetro de campo tipo Philip-Dunne, Slug Test e Poço. Em laboratório são utilizados o Permeâmetro de Carga Variável e o Permeâmetro de Carga Constante.

É comum, no meio geotécnico, a existência de trabalhos que ressaltam as diferenças entre valores de coeficiente de permeabilidade quando da determinação em laboratório ou no campo, tanto em amostras compactadas quanto indeformadas (CRUZ, 2004; REGALADO; CARPENA, 2004; BROOKS; BOLL; MCDANIEL, 2004; JACINTHO; 
GUIMARTAES; CARVALHO, 2006). Dentre os fatores que interferem nas diferenças, o principal é a questão da reduzida dimensão da amostra ensaiada em laboratório, onde é difícil avaliar caminhos preferenciais de percolação relacionados a estruturas reliquiares, atividade biológica e heterogeneidade, principalmente quando se trata de solos residuais.

Em alguns casos, diferenças podem ser encontradas quando são utilizados, para um mesmo solo, tipos diferentes de ensaios mesmo que ambos em laboratório ou em campo. Alonso e Vilar (2006) observaram que ensaios em permeâmetro de parede flexível conduzem a valores de coeficiente de permeabilidade superiores aos determinados em permeâmetros de parede rígida, provavelmente em função da maior saturação conseguida por contrapressão em permeâmetros de parede flexível.

Paz-González et al. (2001) verificaram diferenças entre valores de coeficiente de permeabilidade saturada determinados com Guelph e valores calculados a partir de dados de coeficiente de permeabilidade não saturados, para diferentes sucções, obtidos com tensiômetro. Franch, Cardoso Junior e Futai (2006) encontraram diferenças entre os resultados dos ensaios de Infiltrômetro e Permeâmetro de Guelph para o solo de um aterro compactado com textura variando de argilaarenosa a areia-argilosa.

Chappell e Lancaster (2007) ao estudarem a permeabilidade de campo de uma argila sensível por sete diferentes formas, verificaram que fatores como direção de fluxo, dispersão de partículas, perturbação de amostras, anisotropia e erros podem afetar nas determinações de permeabilidade. Dentro deste contexto, o trabalho objetiva a comparação dos valores de coeficiente de permeabilidade do solo argiloso laterítico do CEEG determinado segundo diferentes métodos de laboratório e em campo.

\section{Local de Estudo - CEEG}

O local de estudo é a área do Campo Experimental de Engenharia Geotécnica (CEEG) que se encontra no interior do campus universitário da Universidade Estadual de Londrina (UEL) situada na cidade de Londrina/ PR, localizada ao sul do Brasil, aproximadamente entre a longitude $50^{\circ} 05^{\prime}$ Oeste e a latitude $23^{\circ} 30^{\prime}$ Sul.

De acordo com o Instituto agronômico do Paraná (IAPAR, 2014), a região se caracteriza pelo tipo climático Cfa de Koppen, apresentando clima subtropical. A precipitação média anual se situa na faixa de 1400 a $1600 \mathrm{~mm}$ e a temperatura média anual na faixa de 21 a $22^{\circ} \mathrm{C}$, com média anual mínima na faixa de 16 a $17^{\circ} \mathrm{C}$ e média anual máxima na faixa de 27 a $28^{\circ} \mathrm{C}$. A Geologia predominante no município é a formação Serra Geral, pertencente à Bacia Sedimentar do Paraná e a litologia principal é a rocha vulcânica básica, o basalto (JKsg).

As investigações de campo na área do CEEG, com ensaios SPT, CPT e DMT, realizadas por Teixeira, Mangieri Sobrinho e Branco (2006), indicaram diferentes interpretações para cada tipo de ensaio, cujas localizações estão indicadas na Figura 1. O perfil definido por meio do ensaio SPT foi subdividido em 7 estratos, enquanto que por meio do CPT em 4 estratos e DMT em 6 estratos. Também houve discordâncias entre os resultados quanto à classificação textural. No ensaio SPT, a classificação táctil-visual identifica o solo como argiloso, enquanto que nos ensaios de CPT e DMT, o comportamento do solo indica se tratar de material siltoso. Estas discordâncias são geradas pelo fato dos ensaios CPT e DMT fornecerem a classificação do solo em função do seu comportamento e o SPT em função da granulometria, obtida por meio de análise táctil-visual. A Figura 2 apresenta o perfil do solo, 
Figura 1. Locação dos furos de sondagem no CEEG

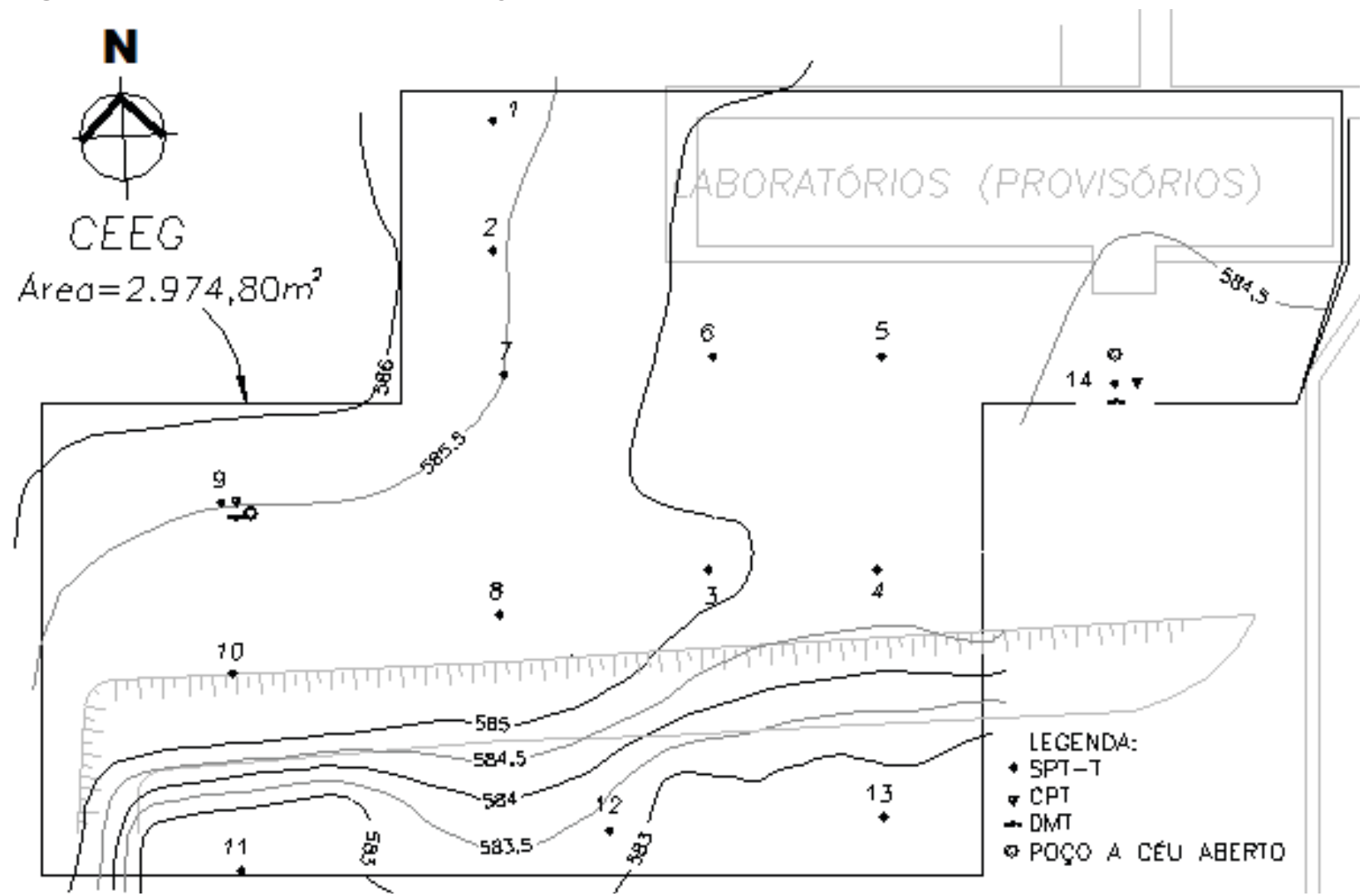

Fonte: Cavalcante et al. (2007).

Figura 2. Perfil de SPT (Leste-Oeste)

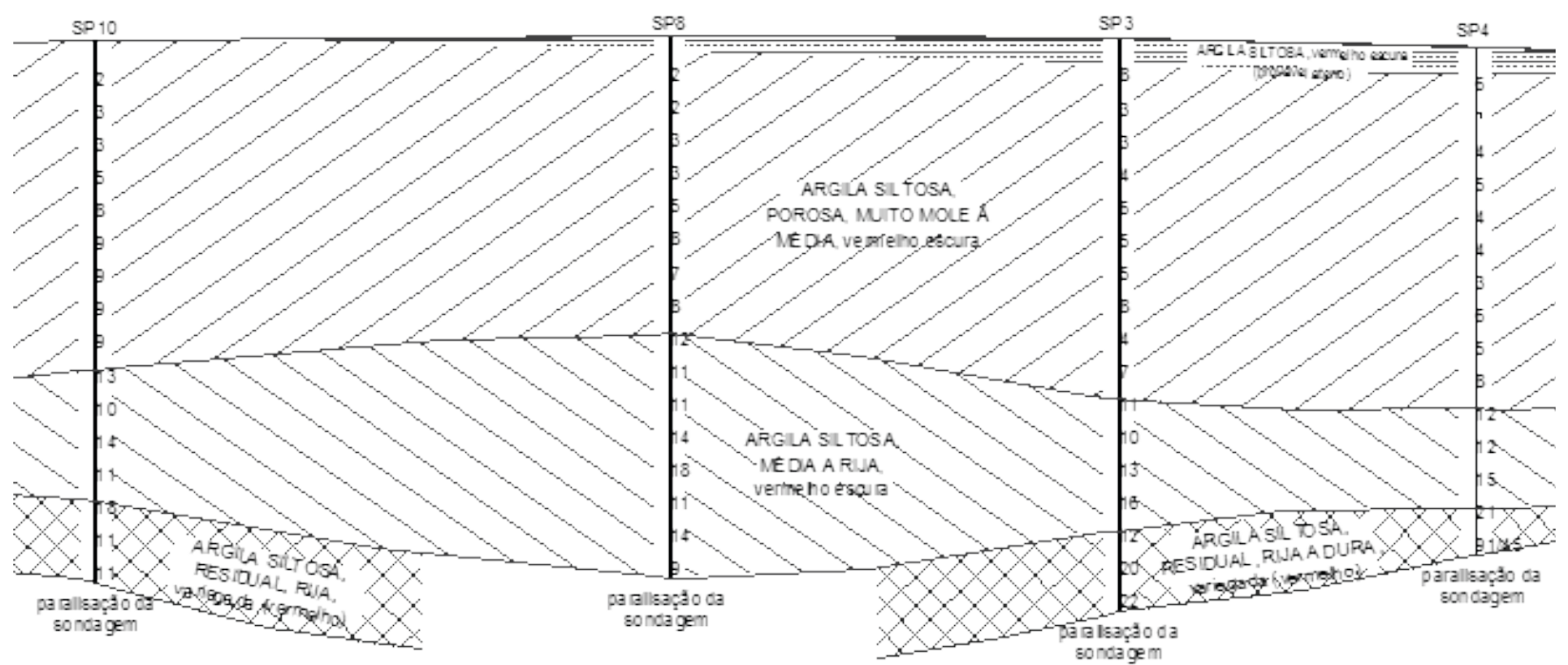

Fonte: Cavalcante et al. (2007). 
O perfil de solo da Figura 2 foi avaliado sob o foco pedológico por Teixeira, Mangieri Sobrinho e Branco (2006), que, considerando o Sistema Brasileiro de Classificação de solos da EMBRAPA (1999), classificaram o perfil do solo da área do CEEG como Latossolo. Segundo estes autores, o resultado do intemperismo sofrido pela rocha basáltica resultou em um perfil de solo composto por: horizonte A, com profundidade em torno de $0,5 \mathrm{~m}$ e com presença de matéria orgânica; horizonte $\mathrm{B}$, espesso, com profundidade máxima de $11 \mathrm{~m}$ e laterizado; e, por último, horizonte $\mathrm{C}$, composto pela alteração de rocha, a alterita, com a presença da estrutura reliquiar do basalto. Ao longo do Horizonte B o solo é vermelho escuro devido à oxidação do ferro e alumínio, apresentando alto teor de argila em torno de $55 \%$, elevado índice de vazios, (em torno de 2), baixo peso específico aparente (em torno de $14 \mathrm{kN} / \mathrm{m}^{3}$ ) e elevado peso específico dos sólidos (em torno de $30 \mathrm{kN} / \mathrm{m}^{3}$ ) (TEIXEIRA; MANGIERI SOBRINHO; BRANCO, 2006).

Os mesmos autores relatam que o solo deste estrato, quando submetido ao ensaio de sedimentação, apresenta um percentual de partículas de argila em torno de $55 \%$ quando se utiliza defloculante (hexametafosfato de sódio + carbonato de sódio). Caso a sedimentação seja feita sem defloculante, a porcentagem de argila decresce a valores inferiores a 5\%. Rocha, Barros e Guimarães (1991) estudaram a composição mineralógica do perfil de latossolo situado no Campus da UEL e identificaram predomínio de caulinita, gibsita, vermiculita e uma importante fração de hematita e goethita (óxidos de ferro).

Miguel, Teixeira e Padilha (2006) em trabalho sobre curva caracterítica de sucção do solo do CEEG verificaram que o solo apresenta comportamento similar a solos arenosos com curvas de sucção semelhantes a de outros solos finos lateríticos. Teixeira et al. (2008) concluíram que as estruturas naturais macroporosas e microporosas dos solos finos lateríticos fazem com que na prática estes solos tenham o comportamento de siltes e até de areias finas.

\section{Ensaios de Laboratorio}

Os ensaios de laboratório foram conduzidos por Weiss (2010) com corpos de prova indeformados obtidos a partir de moldagem de amostras indeformadas. Estas amostras foram obtidas no Campo Experimental de Engenharia e Geotécnica da Universidade Estadual de Londrina (CEEG UEL) em uma cava aberta com dimensões aproximadas de 2,40 ×3,20 me profundidade de 1,90 m, ao lado da sondagem de número 9 (Figura 1). As coletas foram feitas do fundo do poço, na forma de blocos com dimensões de $30 \mathrm{~cm}$ de aresta, segundo as recomendações do DNER (1994). Os corpos de prova, usados em laboratório, foram obtidos por talhagem a apartir dos blocos. Para auxiliar a talhagem, foi criado um molde cilíndrico bipartido de PVC que serviu de guia para a moldagem, garantindo uniformidade das dimensões.

A determinação dos índices físicos foi feita por meio das seguintes correlações descritas nas equações de 1 a 4 (PINTO, 2002).
(1) $e=\frac{\gamma_{s}}{\gamma_{d}}-1$
(2) $S=\frac{\gamma_{s} \cdot w}{\gamma_{w} \cdot e}$
(3) $w=\frac{M_{w}}{M_{s}} \cdot 100$
(4) $\gamma_{d}=\frac{\gamma_{n}}{(1+w)}$

Onde,

$\gamma \mathrm{s}=$ massa específica dos sólidos $\left(\mathrm{g} / \mathrm{cm}^{3}\right)$

$\gamma \mathrm{d}=$ massa específica seca do solo

$\gamma \mathrm{n}=$ massa específica natural do solo

$\mathrm{e}=$ índice de vazios

$\mathrm{S}=$ grau de saturação

$\mathrm{W}=$ umidade $(\%)$ 
$\mathrm{Mw}=$ massa de água

Ms = massa de sólidos

\section{Permeâmetro de Carga Variável}

O ensaio no permeâmetro de carga variável foi realizado segundo o método "B" da ABNT (2000). Este ensaio é comumente utilizado quando o coeficiente de permeabilidade é muito baixo (mais usado em solos argilosos), pois a determinação pelo permeâmetro de carga constante é pouco precisa (PINTO, 2002). É utilizada a diferença entre as cargas em um determinado intervalo de tempo. Verifica-se o tempo que a água da bureta leva para baixar da altura inicial "Hi" para a altura final "Hf" num instante " $\mathrm{t}$ " qualquer. O coeficiente de permeabilidade é obtido pela Equação 5.

$$
k=\frac{a \cdot L}{t \cdot A} \cdot \log \left(\frac{H_{i}}{H_{f}}\right)
$$

Onde:

$$
\mathrm{H}_{\mathrm{i}}=\text { carga total inicial no tempo } t
$$

$\mathrm{H}_{\mathrm{f}}=$ carga total fina no tempo $t$

$\mathrm{a}=$ área da seção transversal da proveta

$\mathrm{L}=$ comprimento de percolação (altura do corpo de prova)

$\mathrm{A}$ = área da seção transversal à direção de fluxo (área da seção transversal do corpo-de-prova)

$t=$ tempo determinado entre as leituras de $\mathrm{H}_{\mathrm{i}} \mathrm{eH}_{\mathrm{f}}$

\section{Permeametro de Carga Constante}

O ensaio no permeâmetro de carga constante foi realizado segundo a ABNT (1995). Este ensaio é indicado para solos arenosos e consiste na manutenção da carga "H" e medição do volume da água percolado e do tempo de percolação correspondente. Assim, conhecidas as vazões e as características geométricas, o coeficiente de permeabilidade é calculado segundo a Equação 6.

$$
k=\frac{Q \cdot L \cdot}{\Delta H \cdot A}
$$

Onde,

$\mathrm{Q}$ = vazão (relação entre o volume de água percolada e o tempo de percolação $\mathrm{em} \mathrm{m}^{3} / \mathrm{s}$ )

$\mathrm{k}=$ coeficiente de permeabilidade $(\mathrm{m} / \mathrm{s})$

$\Delta \mathrm{H}=$ perda de carga do sistema $(\mathrm{m})$

$\mathrm{L}=$ comprimento da amostra $(\mathrm{m})$

$\mathrm{A}=$ área da amostra perpendicular ao fluxo $\left(\mathrm{m}^{2}\right)$

\section{Ensaios In Situ}

Os ensaios in situ foram realizados por Weiss (2010) no fundo da cava, ao lado da área de coleta das amostras indeformadas, garantindo que a profundidade de ensaio seja a mesma tanto para os ensaios de laboratório quanto para os de campo.

\section{Infiltrômetro}

O infiltrômetro utilizado é composto por dois cilindros metálicos de $0,2 \mathrm{~cm}$ de espessura e $15,0 \mathrm{~cm}$ de altura, com diâmetros de $12,0 \mathrm{~cm}$ e $24,0 \mathrm{~cm}$, com as bordas inferiores mais finas em forma de bisel para facilitar a penetração no solo, causando o mínimo de desestruturação. Os cilindros concêntricos são cravados à percussão com auxílio de marreta.

$\mathrm{O}$ cilindro interno funciona como reservatório e apresenta régua graduada para leitura da altura de água, nos tempos de 1, 2, 3, 4, 5, 10, 20, 30, 45, 60, 90 e 120 minutos, com a finalidade de determinar a infiltração ao longo do tempo. A lâmina de água utilizada foi de $7,5 \mathrm{~cm}$ no cilindro interno e de 5 $\mathrm{cm}$ no externo, conforme indicação de Martins e Paiva (2003). O uso do cilindro externo, também com água, é importante para garantir que a direção 
de fluxo seja predominantemente vertical na região onde a infiltração é medida. $\mathrm{O}$ valor do coeficiente de permeabilidade é determinado pela Equação 7 na condição onde a taxa de infiltração permanece constante.

$$
k=\frac{\Delta h}{\Delta t}
$$

Onde:

$$
\begin{aligned}
& \Delta h=\text { variação de altura da lâmina d'água } \\
& \Delta t=\text { intervalo de tempo }
\end{aligned}
$$

\section{Guelph}

O permeâmetro de Guelph (Figura 6) é composto por: garrafa de Mariotte, que controla a carga constante dentro do furo. O aparelho é composto por um tripé que permite a sua adaptação em terrenos irregulares e também conta com reservatório interno graduado. Este permeâmetro mede a permeabilidade saturada de campo acima do lençol freático (AGUIAR, 2001).

Quando da utilização do equipamento, após algum tempo, uma pequena área em torno do furo estará saturada e então o fluxo se torna constante. Em uma situação de campo não se consegue expulsar completamente o ar dos vazios. Sendo assim, é difícil atingir a saturação total. Com isso, a saturação atingida é uma chamada saturação de campo, porém, isso não chega a ser uma desvantagem (SOUZA, 2007).

Existe mais de uma técnica para utilização do permeâmetro de Guelph: método com aplicação de apenas uma altura carga e método com aplicação de duas alturas de carga. Inicialmente os dois métodos foram testados e não foi observada diferença nos valores válidos obtidos que justificasse o uso do método com duas cargas. Como o método com duas cargas apresentou em alguns casos valores negativos, que seriam desprezados, optou-se por utilizar o método de uma altura de carga. A existência de valores negativos e erráticos no ensaio de duas alturas de carga é comum quando o meio é heterogêneo ou quando é marcante a presença de macroporos (AGUIAR, 2001; GARCIA-SINOVAS et al., 2001).

O princípio do equipamento é de que mantendo uma pequena carga o fluxo atinge rapidamente o regime permanente, a partir de um furo cilíndrico em solo não saturado (BASTOS, 2004). O cálculo da permeabilidade através do método de uma altura de carga segue a Equação 8 e depende do parâmetro $\alpha$, referente ao tipo de solo, cujos valores estão apresentados no Quadro 1.

$$
k=\frac{C \cdot Q}{\left[2 \cdot \pi \cdot H^{2}+C \cdot \pi \cdot a^{2}+\left(\frac{2 \cdot \pi \cdot H}{\alpha^{*}}\right)\right]}
$$

Onde:

$$
\mathrm{Q}=\text { vazão }
$$

$\mathrm{a}=$ raio do furo

$\mathrm{H}=$ carga de pressão

$\mathrm{C}=$ parâmetro obtido do gráfico $\mathrm{CxH} / \mathrm{a}$ (Figura 3)

$\alpha^{*}=$ parâmetro relacionado ao tamanho dos poros

Quadro 1 - Valores de $\alpha$ para diferentes tipos de solo.

\begin{tabular}{|c|c|}
\hline Tamanho de Poros & $\alpha^{\star}\left(\mathrm{m}^{-1}\right)$ \\
\hline $\begin{array}{c}\text { Materiais argilosos compactados e } \\
\text { estruturados }\end{array}$ & 1 \\
\hline $\begin{array}{c}\text { Materiais com grãos finos (argilosos) } \\
\text { e não estruturados }\end{array}$ & 4 \\
\hline Argilas, areias fina e média & 12 \\
\hline $\begin{array}{c}\text { Solos com grãos graúdos e } \\
\text { solos estruturados com fissuras e } \\
\text { macroporos }\end{array}$ & 36 \\
\hline
\end{tabular}

Fonte: Modificado de Aguiar (2001). 
Figura 3. Gráfico C x H/a

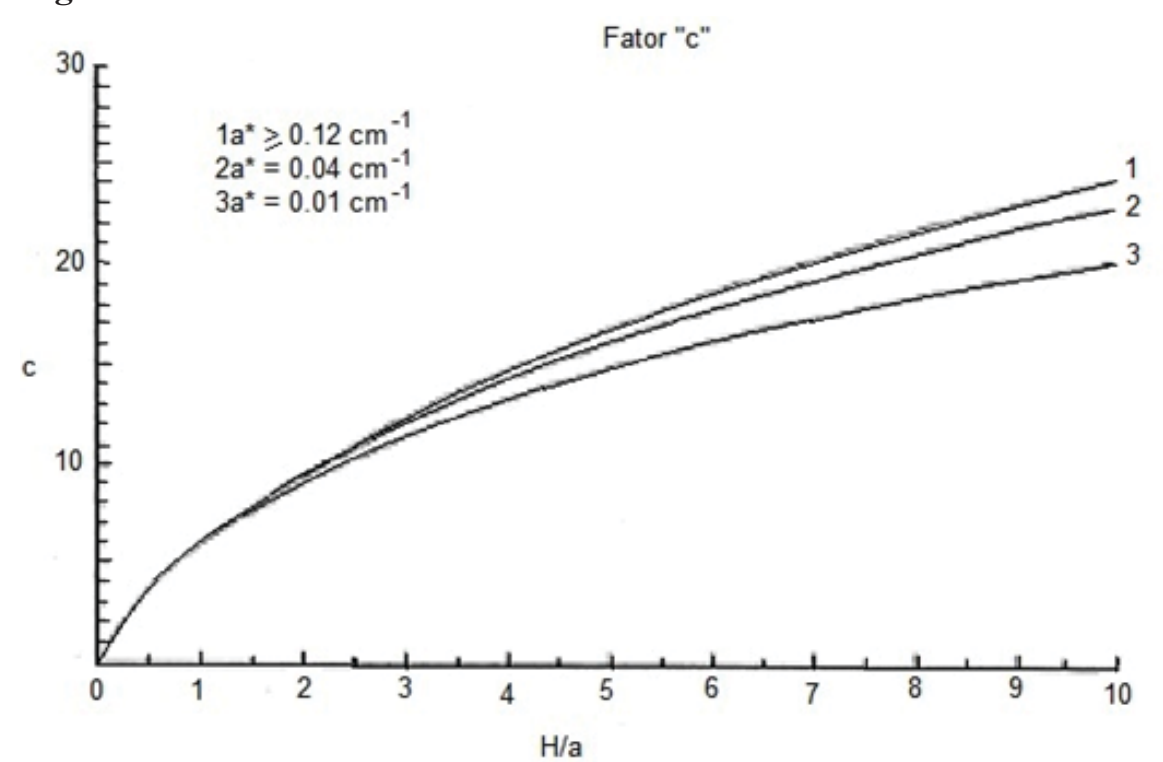

Fonte: Reynolds; Elrick; Topp (1983) apud Aguiar (2001).

\section{Resultados e Análises}

Os resultados dos ensaios em laboratório e em campo, realizados com os diferentes equipamentos, estão apresentados no Quadro 2, onde estão apresentados os valores de coeficiente de permeabilidade obtidos em cada teste, o valor médio para cada tipo de ensaio e o valor do coeficiente de variação em relação à média para cada ensaio. A exceção do resultado do coeficiente de permeabilidade pelo permeâmetro de carga constante, os valores médios de permeabilidade se situaram na ordem de grandeza de $10^{-3} \mathrm{~cm} / \mathrm{s}$, independente do equipamento utilizado. $\mathrm{O}$ valor médio de coeficiente de permeabilidade determinado pelo permeâmetro de carga constante apresentou ordem de grandeza inferior aos dos demais ensaios. Os autores consideram que pode ter havido perda de carga no sistema de alimentação visto que não se pode utilizar permeâmetro com tubos manométricos, o que garantiria medidas de perda de carga apenas no solo.
Quadro 2 - Resultados dos ensaios de laboratório.

\begin{tabular}{|c|c|c|c|c|}
\hline Equipamento & $\mathrm{N}$ & $\mathrm{k}(\mathrm{cm} / \mathrm{s})$ & Média & CV (\%) \\
\hline \multirow{5}{*}{ Constante } & 1 & 0,9E-04 & \multirow{5}{*}{$6,7 \mathrm{E}-04$} & \multirow{5}{*}{92} \\
\hline & 2 & $1,1 \mathrm{E}-04$ & & \\
\hline & 3 & 7,3E-04 & & \\
\hline & 4 & $15,8 \mathrm{E}-04$ & & \\
\hline & 5 & $8,3 \mathrm{E}-04$ & & \\
\hline \multirow{4}{*}{ Variável } & 1 & $0,8 \mathrm{E}-03$ & \multirow{4}{*}{$3,5 \mathrm{E}-03$} & \multirow{4}{*}{80} \\
\hline & 2 & $2,4 \mathrm{E}-03$ & & \\
\hline & 3 & $3,5 \mathrm{E}-03$ & & \\
\hline & 4 & 7,4E-03 & & \\
\hline \multirow{4}{*}{ Infiltrômetro } & 1 & 8.3E-03 & \multirow{4}{*}{$5,8 \mathrm{E}-03$} & \multirow{4}{*}{37} \\
\hline & 2 & $6.7 \mathrm{E}-03$ & & \\
\hline & 3 & $5.0 \mathrm{E}-03$ & & \\
\hline & 4 & $3.3 \mathrm{E}-03$ & & \\
\hline $\begin{array}{c}\text { Guelph } \\
\alpha^{\star}=0,12\end{array}$ & 1 & 3.7E-04 & $1,3 \mathrm{E}-03$ & 76 \\
\hline
\end{tabular}

Fonte: Elaboração dos Autores

Uma das dificuldades dos cálculos da permeabilidade nos ensaios com permeâmetro de Guelph foi a escolha do parâmetro $\alpha$ dentre as quatro possibilidades apresentadas no Quadro 1. 
Os valores de 12 ou 36 poderiam ser justificados, considerando a granulometria (argila siltosa/silte argiloso) e a existência de poros visíveis a olho nu. $\mathrm{O}$ valor médio de $\mathrm{k}$ obtido nos cálculo foi de 1,3×10${ }^{3} \mathrm{~cm} / \mathrm{s}$, para $\alpha$ igual a 12 . Caso fosse adotado o valor de 36, a média praticamente dobraria atingindo o valor de $2,5 \times 10^{-3} \mathrm{~cm} / \mathrm{s}$. Para prática de engenharia geotécnica, a diferença não é significativa, pois usualmente se trabalha com ordem de grandeza do coeficiente de permeabilidade, podendo ser considerada como $10^{-3} \mathrm{~cm} / \mathrm{s}$. Regalado \& Carpena (2004) também verificaram que o erro na escolha de $\alpha$ pode ser desconsiderado.

Valores de $\mathrm{k}$ da ordem de grandeza $10^{-3} \mathrm{~cm} / \mathrm{s}$ para o solo do CEEG foram também encontrados por Teixeira, Mangieri Sobrinho e Branco (2006) em pesquisas anteriores realizadas dentro do campus da UEL em outras profundidades, porém dentro do mesmo extrato de solo classificado como argila siltosa porosa muito mole à média (Figura 2). Cavalcante et al. (2007), no relato sobre Campus Experimentais Brasileiros, mostram para o solo do campo experimental da UEL, valor de coeficiente de permeabilidade de $3,31 \times 10^{-3} \mathrm{~cm} / \mathrm{s}$ obtido na profundidade de um metro por ensaio de infiltração, determinado conforme ABGE (1996).

Roque (2009) obteve em local próximo, no mesmo extrato de solo, valor médio de $3,5 \times 10^{-3} \mathrm{~cm} / \mathrm{s}$ em nove ensaios com infiltrômetro na profundidade de $0,10 \mathrm{~m}$. Vale ressaltar que o valor de coeficiente de permeabilidade médio obtido apresentou-se alto se for considerada a classificação granulométrica de argila siltosa sedimentar, onde a faixa esperada é da ordem de $10^{-6}$ a $10^{-7} \mathrm{~cm} / \mathrm{s}$ (PINTO, 2002).

Os coeficientes de variação $(\mathrm{CV})$ encontrados nos ensaios de permeabilidade (Quadro 2) variaram de $37 \%$ a $92 \%$. Os valores de CV encontrados são considerados altos em análises estatísticas (valores maiores que 30\%), indicando que a variabilidade em relação à média é alta (FONSECA; MARTINS, 2008). Contudo, valores elevados de coeficiente de variação em ensaios de permeabilidade tanto de campo como de laboratório têm sido reportados na literatura e são esperados (GARCIA-SINOVAS et al., 2001; PAZ GONZALES et al., 2001; SÃO MATEUS; MAVHADO; BARBOSA, 2012). Analisando os valores de CV, pode se dizer que o ensaio com infiltrômetro indicou menor variabilidade que os demais.

O grau de saturação final do ensaio e a porosidade são comumente citados na literatura como fatores condicionantes do coeficiente de permeabilidade. Scherpinski et al. (2010) consideraram a porosidade como sendo o possível fator de variação da permeabilidade determinada com Guelph em latossolo vermelho distroférrico. Apesar de ser comum relacionar coeficiente de permeabilidade com porosidade, os autores da área de Geotecnia costumam relacionar o coeficiente de permeabilidade com o índice de vazios, principalmente quando se trata de análises ao longo de um processo de adensamento.

Na busca de avaliar a influência de uma possível interferência da representatividade das amostras de laboratório, realizou-se o cálculo das médias e coeficientes de variação do índice de vazios e grau de saturação final das amostras utilizadas para os ensaios com permeâmetro de carga constante e permeâmetro de carga variável (Quadro 3). Os baixos valores de coeficiente de variação demonstram que os valores de índice de vazios e grau de saturação final não contribuem, de forma significativa, para a variação do coeficiente de permeabilidade.

Quadro 3- Resultados dos ensaios de laboratório.

\begin{tabular}{|c|c|c|c|c|}
\hline Equipamento & \multicolumn{2}{|c|}{ Constante } & \multicolumn{2}{c|}{ Variável } \\
\hline Índices & $\mathrm{e}$ & $\mathrm{S}(\%)$ & $\mathrm{e}$ & $\mathrm{S}(\%)$ \\
\hline Ensaio 1 & 2,08 & 78 & 2,12 & 82 \\
\hline Ensaio 2 & 1,95 & 96 & 1,93 & 92 \\
\hline Ensaio 3 & 1,82 & 99 & 1,85 & 96 \\
\hline Ensaio 4 & 2,18 & 91 & 2,19 & 92 \\
\hline Ensaio 5 & 2,13 & 91 & - & - \\
\hline Media & 2,03 & 91 & 2,02 & 90 \\
\hline CV (\%) & 7 & 9 & 8 & 7 \\
\hline
\end{tabular}

Fonte: Elaboração dos Autores 
Com o intuito de determinar uma possível correlação entre os índices físicos e valores de coeficiente de permeabilidade, como a relação de Kozeny-Carman utilizada por Regalado \& Carpena (2004) ou a encontrada por Moraes Silva (2000), foram analisadas, para cada permeâmetro, as relações entre índice de vazios e coeficiente de permeabilidade (Figura 4) e grau de saturação e coeficiente de permeabilidade (Figura 5).

Com respeito ao índice de vazios (Figura 4), os coeficiente de determinação $\left(\mathrm{R}^{2}\right)$ encontrados foram de 0,08 para carga constante e de 0,33 para carga variável. levando a coeficiente de correlação de Pearson (R) de 0,28 e 0,58, respectivamente.
Com relação ao grau de saturação (Figura 5), os coeficientes de determinação $\left(\mathrm{R}^{2}\right)$ foram de 0,19 para carga constante e de 0,13 para carga variável, culminando em coeficientes de correlação de Pearson $(\mathrm{R})$ de 0,43 e 0,36 respectivamente.

As correlações podem ser classificadas, segundo Bisquerra, Castella e Marinez (2004), como baixa a moderada. Dessa forma, contrariando o esperado teoricamente, não foi encontrada uma correlação que suporte a teoria clássica de aumento de valor de coeficiente de permeabilidade com aumento de índice de vazios e aumento de grau de saturação.

Figura 4. Índice de vazios (e) versus permeabilidade (k) para os ensaios de laboratório.

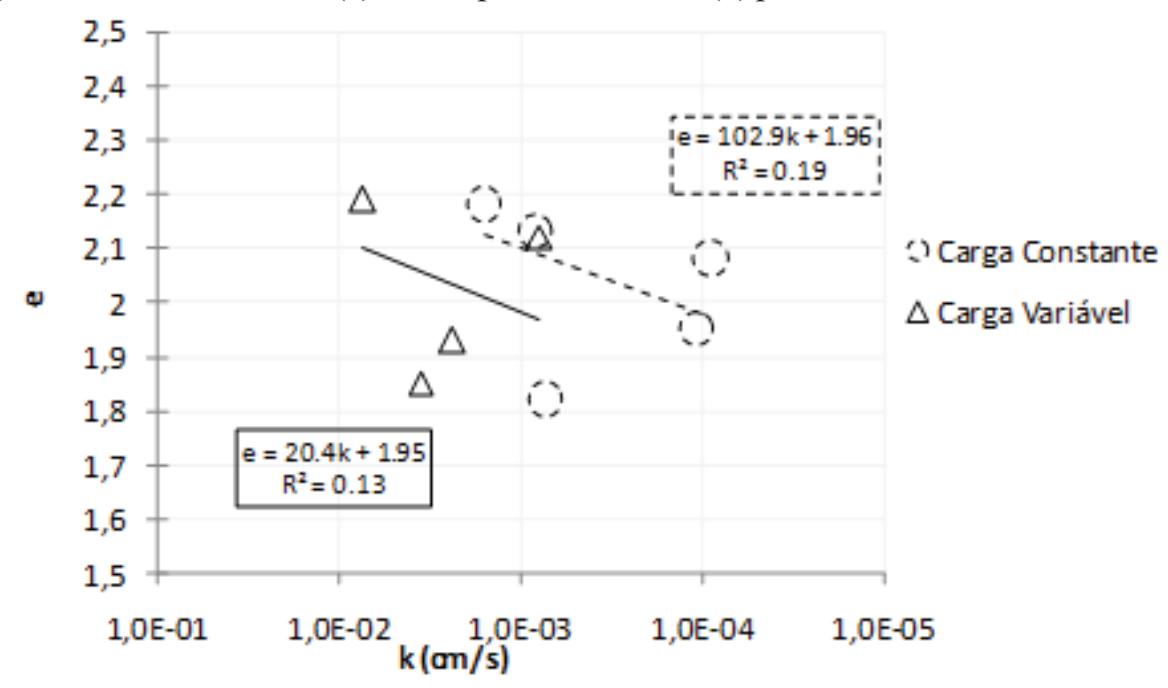

Fonte: Elaboração dos Autores

Figura 5. Grau de Saturação(S) versus permeabilidade (k) para os ensaios de laboratório.

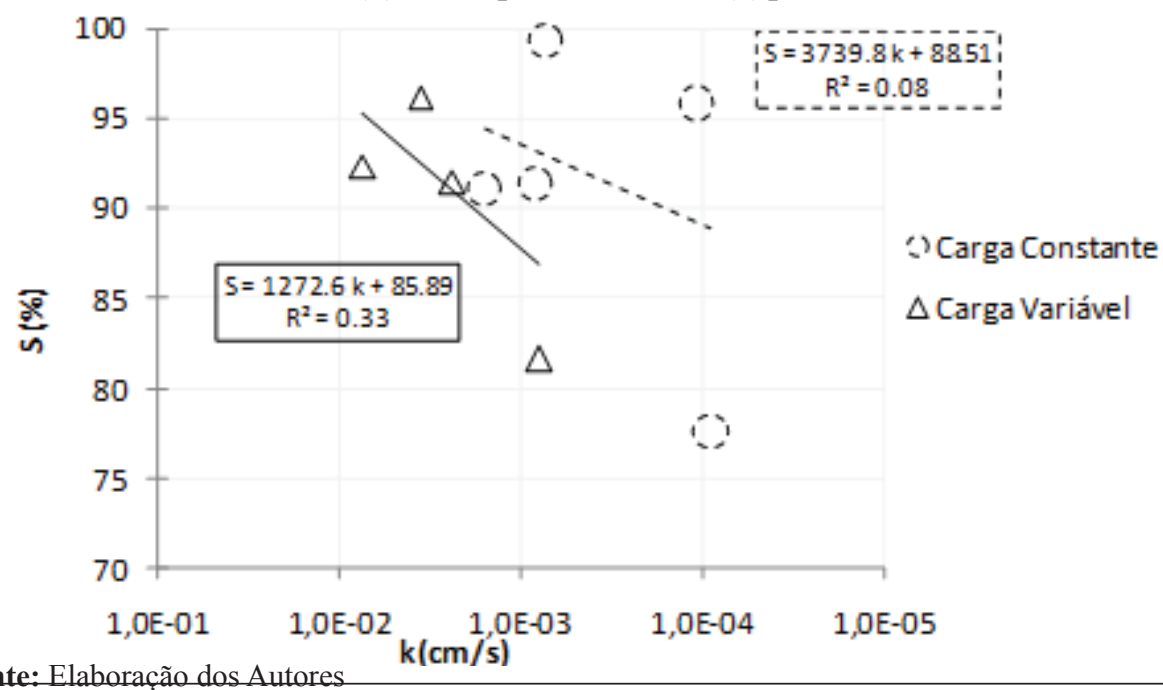


Através das diversas análises realizadas, podese observar que: (1) o valor médio do coeficiente de permeabilidade não é compatível com a granulometria dispersa, ou seja, com defloculante; (2) as amostras não apresentam heterogeneidade quanto a índice de vazios e grau de saturação final que justifique as dispersões nos ensaios; e (3) não foi possível obter correlações satisfatórias entre índice de vazios e coeficientes de permeabilidade nos ensaios de laboratório.

A observação 1 ocorre em função da estrutura do solo estudado que apresenta a formação de microagregações de tamanho silte e silte/argila, conduzindo a estrutura mais porosa do que a esperada para um solo argiloso. As indicações de tal comportamento foram avaliadas nos estudos de Teixeira et al. (2004), que verificaram diferenças marcantes entre a distribuição granulométrica do solo obtida com e sem uso de defloculante. E, ainda, corroboradas nos estudos de Teixeira et al. (2008) que concluíram, na determinação da análise da estratigrafia obtida por meio de investigação com CPT e DMT, que o solo do CEEG pode ser identificado em campo como silte e areia fina em função da agregação de partículas finas. Este tipo de comportamento é comumente relatado por diversos autores em estudos sobre solos lateríticos finos (RODRIGUEZ, 2005; LACERDA, 2010).

A observação 2 contradiz o esperado teoricamente, visto que amostras consideradas homogêneas em termos de grau de saturação final e índice de vazios (ou porosidade) resultaram em alto coeficiente de variação. A explicação para este fato é de que os índices físicos são determinados a partir de valores globais da amostra (massa total e volume total do corpo de prova) e podem não estar correlacionados ao arranjo estrutural do solo que pode ser diferente para cada amostra.

A observação 3 complementa a observação 2 e mostra que não há correlação satisfatória entre índice de vazios e coeficiente de permeabilidade para os resultados deste trabalho. Considerando a possibilidade de arranjos estruturais distintos, dois condicionantes podem estar relacionados: macroporosidade e vazios não conectados. Olivella e Gens (2000), ao estudarem argilas expansivas, observaram que a mudança de umidade na amostra gera alteração dos macroporos que são os controladores do coeficiente de permeabilidade. Logo, as correlações com o índice de vazios só ocorreria se as diferenças de índice de vazios representassem arranjos estruturais similares com menor quantidade de macroporos, como o que ocorre em determinações de coeficiente de permeabilidade durante ensaio de adensamento (SILVA, 2000).

A consideração da existência de vazios não conectados e o controle de fluxo e permeabilidade pelos vazios interconectados são conceitos utilizados no estudo de aqüíferos. Trabalhos que tratam deste assunto determinam a permeabilidade a partir de um parâmetro denominado de porosidade efetiva, que é a relação entre vazios interconectados (por onde passa o fluxo) e o volume total do material e que pode ser determinada com traçadores por ensaios de campo ou de laboratório (HALL; LUFTRELL; CRONIN, 2001; STEPHENS et al., 1998; VERMA, 2011). Dessa forma, os autores sugerem para trabalhos futuros o estudo da porosidade efetiva do solo do CEEG e da relação desta com a permeabilidade saturada de solo.

\section{Conclusões}

Não há diferença na ordem de grandeza do valor do coeficiente de permeabilidade do solo ensaiado com os equipamentos de permeâmetro de carga variável, infiltrômetro e permeâmetro de Guelph. O valor médio de coeficiente de permeabilidade determinado pelo permeâmetro de carga constante apresentou ordem de grandeza inferior aos dos demais ensaios, porém pode ser devido ao sistema utilizado.

Os coeficientes de variação encontrados para os ensaios são altos (de 37\% a 92\%), mostrando a alta variabilidade em relação à média. Contudo, 
encontram-se na faixa de valores usualmente encontrada na literatura nacional e internacional mesmo para areias. Este é um dos fatores que leva à majoração do valor do coeficiente de permeabilidade em 5 a 10 vezes para determinação do coeficientye de permeabilidade de projeto (CRUZ, 2004).

Considerando que as determinações de coeficiente de permeabilidade obtidas neste trabalho estão compatíveis com determinações de estudos anteriores realizados em outras profundidades, a faixa de valores médios de coeficiente de permeabilidade encontrados para o solo do CEEG, da ordem de $10^{-3} \mathrm{~cm} / \mathrm{s}$, pode ser considerada representativa para o extrato superior formado por argila siltosa porosa e ser adotada em projetos preliminares de engenharia.

$\mathrm{O}$ estudo indica que o solo deve apresentar heterogeneidade com respeito à estrutura do solo laterítico (macro e microporos), não identificada através da análise de índice de vazios, e que esta provavelmente é o principal fator controlador do coeficiente de permeabilidade. Sugere-se estudo sobre a porosidade efetiva do solo do CEEG na busca de confirmar esta hipótese.

\section{Referências}

ASSOCIAÇÃO BRASILEIRA DE GEOLOGIA DE ENGENHARIA E AMBIENTAL - ABGE. Ensaios de permeabilidades em solos: orientações para sua execução no campo. São Paulo: ABGE, 1996. (Boletim, n. 4).

ASSOCIAÇÃO BRASILEIRA DE NORMAS TÉCNICAS - ABNT. NBR 13292: Solo: determinação do coeficiente de permeabilidade de solos granulares à carga constante. Rio de Janeiro, 1995.

ASSOCIAÇÃO BRASILEIRA DE NORMAS TÉCNICAS - ABNT. NBR 14545: Solo: determinação do coeficiente de permeabilidade de solos argilosos a carga variável. Rio de Janeiro, 2000.

AGUIAR, A. B. O Emprego do permeâmetro de Guelph na determinação da permeabilidade do solo, de camadas de lixo e sua cobertura. Rio de Janeiro: COPPE/UFRJ, 2001.
ALONSO, T. P; VILAR, O. M. Condutividade hidráulica em ensaios com permeâmetro de parede flexível vs parede rígida. In: CONGRESSO BRASILEIRO DE MECÂNICA DOS SOLOS E ENGENHARIA GEOTÉCNICA, 13., 2006, Curitiba. Anais... Curitiba, 2006. v. 1, p.161-166.

BARATA, F. E. Algumas considerações criticas sobre "lateritas" e "materiais lateríticos" - necessidade de terminologia brasileira adequada". In.: SIMPÓSIO BRASILEIRO DE SOLOS TROPICAIS EM ENGENHARIA, 1981. Anais... Rio de Janeiro, Universidade Federal do Rio de Janeiro,1981, p.308323.

BASTOS, D. C. O. Manejo da salinidade em irrigação localizada: análise da alternativa de lixiviação incompleta. 2004. Disponível em: $<$ http://www.scribd. com/doc/2535967/Dissertacao-de-Mestrado-DaniloCesar-de-Oliveira-Bastos $>$. Acesso em: 20 ago. 2010.

BIGARELLA, J. J.; BECKER, R. D.; PASSOS, E. Estrutura e origem das paisagens tropicais $e$ subtropicais. Florianópolis: Ed. UFSC, 1996. 2 v.

BISQUERRA, R.; CASTELLA, J.; MARINEZ, F. Introdução a estatística: enfoque informático com o pacote estatístico SPSS. Porto Alegre: ARTMED, 2004.

BRESSANI, L. A., MARTINS F. B., BICA, A. V. D., , Mechanical behaviour of a residual soil from Botucatu sandstone. In: INTERNATIONAL CONGRESS OF IAEG, 7., 1994, Rotterdam. Anais... Rotterdam, Holanda, 1994, v.1, p.315-322.

BROOKS, E. S.; BOLL, J.; MCDANIEL, P.A. A hillslope-scale experiment to measure lateral saturated hydraulic conductivity. Water resources Research, v. 40, W04208, p.1-10, 2004.

CAVALCANTE, E. H.; DANZINGER, F; GIACHETIG, H. L.; COUTINHO, R. Q. Campos Experimentais Brasileiros: campo experimental da UEL, Lisboa. Revista Luso-Brasileira de Geotecnia, n.111, p. 166-177, nov. 2007.

CHAPELL, N. A.; LANCASTER, J. W. Comparison of methodological uncertainties within permeability measurements. Hydrological Processes, n. 21, p. 2504-2514, 2007. 
CRUZ, P. T. 100 barragens brasileiras: oficina de textos. 2. ed. São Paulo: Oficina de Textos, 2004.

DEPARTAMENTO NACIONAL DE ESTRADAS DE RODAGENS - DNER. Procedimento PRO002 - Coletas de amostras indeformadas de solo. DNER, 1994.

EMPRESA BRASILEIRA DE PESQUISA AGROPECUÁRIA - EMBRAPA. Manual brasileiro de classificação de solos: produção de informação. Brasília, 1999.

FONSECA, J. S.; MARTINS, G. A. Curso de estatística. 6. ed. São Paulo: Atlas, 2008.

FRANCH, F. A. J. P.; CARDOSO JUNIOR, C. R.; FUTAI, M. M. Ensaio de infiltração em solo não saturado com medição de poro-pressão da água. In: CONGRESSO BRASILEIRO DE MECÂNICA DOS SOLOS E ENGENHARIA GEOTÉCNICA, 13., 2006, Curitiba. Anais... Curitiba, 2006. v. 2, p. 745-750.

FUTAI, M. M. Estudo teórico-experimental do comportamento de solos tropicais não-saturados: aplicação a um caso de voçorocamento. 2002. Tese (Doutorado em Engenharia Civil) - COPPE/UFRJ, Rio de Janeiro.

GARCIA-SINOVAS, D.; REGALADO C.; MUNOZ-CARPENA, R.; ÁLVAREZ-BENEDI, J. Comparación de los permeámetros de Guelph y Philip-Dunne para la estimación de la condutividad hidráulica saturada del suelo. Temas de Investigación em Zona Saturada. In.: Temas de Investigación en Zona no Saturada., 2001, v. 5. Pamplona, Espanha. Disponível em: < http://abe.ufl.edu/carpena/files/ pdf/zona_no_saturada/temas_de_investigacion_ v5/9.pdf $>$

HALL, S. H.; LUFTRELL, S. P.; CRONIN, W. E. A method for estimating effective porosity and ground-wter velocity. Groundwater, v. 29, n. 2, p. 171-174, March-April, 1991.

INSTITUO AGRONÔMICO DO PARANÁ IAPAR. Cartas Climáticas do Paraná. 2014. Disponível em: <www.iapar.br/modules/conteudo/ conteudo.php? conteudo $=677>$. Acesso em: 20 ago. 2010 .
JACINTHO, E. C.; GUIMARTAES, R. C.; CARVALHO, J. C. de. Estudo da permeabilidade dos solos saprolíticos compactados em campo e em laboratório. In: CONGRESSO BRASILEIRO DE MECÂNICA DOS SOLOS E ENGENHARIA GEOTÉCNICA, 13., 2006, Curitiba. Anais... Curitiba, 2006. v. 1, p. 155-160.

LACERDA, W. A. Shear strength of soils derived from the weathering of granite and gneiss in Brazil. In: CALCATERRA, D.; PARISE, M. (Ed.). Weathering as a predisposing factor to slope movements. London : Geological Society, 2010. (Engineering Geology Special Publications, 23). p. 167-182.

LEROUEIL, S.; VAUGHAN, P. R. The general and congruent effects of structure in natural soils and weak rocks. Geotechnique, London, n. 3, p. 476-488, 1990.

MACCARINI, M.; TEIXEIRA, V. H.; TRICHÊS, G. Bonding properties of a residual soil derived from diabase. In: XII International Conference on Soil Mechanics and Foundation Engineering - XII ICSMFE, 1989, Rio de Janeiro, RJ, Brasil. Anais... Rio de Janeiro, 1989. v. 1, p. 525-528.

MARTINS, E. S. P. R.; PAIVA, J. B. D. Hidrologia aplicada a gestão de pequenas bacias hidrográficas. Porto Alegre: ABRH, 2003.

MASSEY, J. B.; IRFAN, T. Y.; CIPULLO, A. The caracterization of granitic saprolitic soils. In: XII International Conference on Soil Mechanics and Foundation Engineering - XII ICSMFE, 1989, Rio de Janeiro, RJ, Brasil. Anais... Rio de Janeiro, 1989. v. 6, p. 533-542.

MENEZES, S. M.; RIBEIRO K. D.; SAMPAIO F. M. T. Influência da distribuição de vazios na condutividade hidráulica do solo saturado. In: CONGRESSO BRASILEIRO DE MECÂNICA DOS SOLOS E ENGENHARIA GEOTÉCNICA, 13., 2006, Curitiba. Anais... Curitiba, 2006. v. 1, p. 143-148, 2006.

MIGUEL, M. G.; TEIXEIRA, R. S.; PADILHA, A. C. C. Curvas características de sucção do solo laterítico da região de Londrina/PR. Revista de Ciência e Tecnologia, v. 24, n. 12, p. 63- 74, 2006. (RCT24). 
MITCHELL, J. K.; SITAR, N. Engineering properties of tropical residual soils, Proceedings of Specialty Conference on Engineering and Construction in Tropical and Residual Soils, ASCE, Honolulu, p.30-57, 1982.

SILVA, T. R. M. Caracterização e Erodibilidade dos Solos de uma Voçoroca da Região de Ouro Preto, MG. 2000. Dissertação (Mestrado em Engenharia Civil) COPPE/UFRJ, Rio de Janeiro.

NOGAMI, J. S.; VILLIBOR, D. F. Uma nova classificação de solos para finalidades rodoviárias. In: PROC. SIMPÓSIO BRASILEIRO DE SOLOS TROPICAIS EM ENGENHARIA, COPPE/UFRJ, Rio de Janeiro. 1981, v. 1, p. 30-41.

OLIVELLA, S.; GENS, A. Vapour transport in a low permeability unsaturated spoils with capillary effects. Transport in porous media, n. 40, p. 219-241, 2000.

PAZ-GONZÁLEZ, A.; THONON, I.; BERTOLANI, F. C.; TABOADA CASTRO, M. M.; VIDAL VAZQUEZ, E.; DAFONTE, J. Variabilidad espacial de La infiltración en una ladera determinada com permeámetro de Guelph y infiltrómetro de tensión. In.: Temas de Investigación en Zona no Saturada., 2001, v. 5. Pamplona, Espanha. Disponível em: http:/abe.ufl.edu/carpena/files/pdf/ zona_no_saturada/temas_de_investigacion_v5/12.pdf.

PINTO, C. S. Curso básico de mecânica dos solos: com exercícios resolvidos. 2. ed. São Paulo: Oficina de Textos, 2002.

REGALADO, C. M.; CARPENA, R. M. Estimating the saturated hydraulic conductivity in a spatially variable soil with different permeameters: a stochastic Kozeny-Carman relation. Soil Tillage Research, n. 77, p. 189-202, 2004.

REYNOLDS, W. D.; ELRICK, D. E.; TOPP, G. C. $A$ reexamination of the constant head well permeameter method for measuring saturated hydraulic conductivity above the water table. 1983. Soil Science, v. 136, n.4, p.250-268.

ROCHA, G. C.; BARROS, O. N. F.; GUIMARÃES, M. F. Distribuição espacial e características dos solos do Campus da Universidade Estadual de Londrina, PR. Semina: Ciências Agrárias, Londrina, v. 12, n. 1, p.2125, 1991.
RODRIGUEZ, T. T. Proposta de classificação geotécnica para colúvios brasileiros. 2005. Tese (Doutorado em Engenharia Civil) - COPPE/UFRJ, Rio de Janeiro.

ROQUE, G. S. Análise dos parâmetros de infiltração de água no solo do campus da universidade estadual de Londrina - Pr, voltados para aplicação em trincheiras de infiltração. 2009. Trabalho de Conclusão de Curso. (Graduação em Engenharia Civil) - Universidade Estadual de Londrina, Londrina.

SANDRONI, S. S. Sampling and testing of residual soils in Brazil. In: BRAND, E. W.; PHILLIPSON, H. B. Sampling and testing of residual soils: a review of international practice. Hong Kong: Scorpion Press, 1985. p.31-50.

SÃO MATEUS, M. S. C.; MAVHADO, S. L.; BARBOSA, M. C. Na attempt to perform water balance in a Brazilian municipal solid waste landfill. Waste Management, n. 32, p. 471-481, 2012.

SCHERPINSKI, C.; URIBE-OPAZO, M. A.; BOAS, M. A.V.; SAMPAIO, S. C.; JOAHANN, J. A. Variabilidade espacial da condutividade hidráulica e da infiltração da água no solo. Acta Scenarium. Agronomy, Maringá, v. 32, n. 1, p. 7-13, 2010.

SOUZA, R. V. B. Monitoramento hidrogeológico como ferramenta de avaliação à degradação dos recursos hídricos: estudo realizado no aterro controlado de Londrina - PR. Londrina: UEL, 2007.

STEPHENS, D. B.; HSU, K.; PRIEKSAT, M. A.; ANKENY, M. D.; BLANDFORD, N.; ROTH, T. L.; KELSEY, J. A.; WHITWORTH, J. R. A comparison of estimated and calculated effective porosity. Hydrogeology Journal, n. 6, p. 156-165, 1998.

TEIXEIRA, R. S.; BELINCANTA, A.; LOPES, F. F.; GUTIERREZ, N.; BRANCO, C. J. M. C. Avaliação do colpaso do solo da camada superficial da cidade de Londrina/PR. In: SIMPÓSIO BRASILEIRO DE SOLOS NÃO SATURADOS, 5., 2004, São Carlos. Anais... São Carlos: Orencio Monje Vilar, 2004. v.1, p. 495-500. 
TEIXEIRA, R. S.; MANGIERI SOBRINHO V. R.; BRANCO, C. J. M. C. Avaliação do perfil estratigráfico obtido por meio de SPTS, CPTS E DMTS executados em solo laterítico da cidade de Londrina/PR. In: CONGRESSO BRASILEIRO DE MECÂNICA DOS SOLOS E ENGENHARIA GEOTÉCNICA, 13., 2006. Anais... Curitiba, 2006. v. 2 , p. $589-594$.

TEIXEIRA, R. S.; BRANCO, C. J. M. C.; MANGIERI SOBRINHO, V. R.; TEIXEIRA, S. H. C. Avaliação de parâmetros geotécnicos por meio de correlações de resultados de SPT, CPT e DMT. In: CONGRESSO BRASILEIRO DE MECÂNICA DO SOLOS E ENGENHARIA GEOTÉCNICA, 2008. Anais... Búzios-RJ, ABMS, 2008. p.12981305.

VARGAS, M. Identification and classification of tropical soils. US/Brazil. In: GEOTECHNICAL WORKSHOP ON APPLICABILITY OF CLASSICAL SOIL MECHANICS PRINCIPLES TO STRUCTURED SOILS, 23-25, Nov., 1992. p. 200-205.

VERMA, S. Estimation of effective porosity and saturation volume by extended elastic impedance approach: a case study. In: SECOND SOUTH ASIAN.GEOSCIENCE CONFERENCE AND EXHIBITION, 2011, Geoindia, Gearter Noida, New Delhi, India. Resumo Expandido.

WEISS, L. A. Comparação de métodos de permeabilidade no campus da UEL. 2010. Trabalho de Conclusão de Curso. (Graduação em Engenharia Civil) - Universidade Estadual de Londrina, Londrina. 
